\title{
Perspectival Instruments
}

\author{
Ana-Maria Creţu \\ Department of Philosophy, University of Bristol, Bristol, UK \\ Email: d.cretuanamaria@gmail.com
}

(Received 30 October 2020; revised 26 February 2021; accepted 13 April 2021; first published online 12 January 2022)

\begin{abstract}
Despite its potential implications for the objectivity of scientific knowledge, the claim that "scientific instruments are perspectival" has received little critical attention. I show that this claim is best understood as highlighting the dependence of instruments on different perspectives. When closely analyzed, instead of constituting a novel epistemic challenge, this dependence can be exploited to mount novel strategies for resolving two old epistemic problems: conceptual relativism and theory-ladeness. The novel content of this article consists in articulating and developing these strategies by introducing two fine-grained notions of perspectives as the key units of analysis: "broad perspectives" and "narrow perspectives."
\end{abstract}

\section{Introduction}

A scientific instrument is any apparatus, simple or complex, used to investigate the natural world through observation and experimentation. ${ }^{1}$ For example, microscopes, cloud chambers, and spectrographs are all scientific instruments through which we can investigate the world. According to the traditional view, the results of investigations undertaken with scientific instruments are not only invaluable for understanding the world around us, but such results are also objective-call this the objective view of scientific instruments.

Opposed to the objective view are two related theses. The first thesis is the theoryladeness thesis, according to which the background theories, beliefs, or presuppositions of an observer, that is, their perspective, may affect their observations (Hanson 1958). The second is the conceptual relativism thesis, according to which there is no ready world that all perspectives can latch onto; instead, different perspectives construct the world differently (Kuhn 1962). Both theses represent problematic epistemic challenges in relation to the objective view of scientific instruments. Although both problems have been extensively discussed in the literature in relation

\footnotetext{
${ }^{1}$ For historical accounts of instruments, see Turner (2013), McConnell (2013), Brenni (2013), and Hackman (1989). For a conceptual classification, see Lauwerys (1937). For definitional discussions on instruments, see Warner (1990) and Taub (2019).

(C) The Author(s), 2022. Published by Cambridge University Press on behalf of the Philosophy of Science Association. This is an Open Access article, distributed under the terms of the Creative Commons Attribution licence (http:// creativecommons.org/licenses/by/4.0/), which permits unrestricted re-use, distribution and reproduction, provided the original article is properly cited.
} 
to observation and experimentation, comparatively few discussions have focused specifically on scientific instruments, with the notable exception of Chalmers (2003), Heidelberger (2003), and Baird (2004). ${ }^{2}$

In recent years, perspectival realism has emerged as a new view in the philosophy of science, opposed to the objective view. Perspectival realism can be understood as a series of views equally committed to the mind-independence of the world and to the situatedness or perspectivity of scientific knowledge (cf. Massimi 2018c; Teller 2011; Giere 2006). ${ }^{3}$ As defended by Giere $(1999,2000,2006)$, and recently by Evans (2020), perspectival realism holds that scientific instruments are perspectival. ${ }^{4}$ I will show that this claim is best understood as highlighting the dependence of instruments on different perspectives. When closely analyzed, instead of constituting a novel epistemic challenge, this dependence can be exploited to mount novel strategies for resolving two old epistemic problems: conceptual relativism and theory-ladeness. The novel content of this article consists in articulating and developing these strategies by introducing two fine-grained notions of perspectives as the key units of analysis: "broad perspectives" and "narrow perspectives."

The first strategy is developed to respond to the conceptual relativism brought about by "instrumental incommensurability," understood as a form of discontinuity or incommensurability at the level of the instruments themselves. The strategy is developed around broad perspectives, understood as culturally and scientifically situated theoretical frameworks typical of a scientific community (Massimi 2018c). I show that far from constituting a novel epistemic challenge, the dependence of instruments on broad perspectives, when closely analyzed, provides instead the means to mount a novel response to the old epistemic challenge of conceptual relativism.

By recourse to the history and philosophy of science, three points are established. First, it is established that objectivity standards (cf. Daston and Galison 2007)—which govern the use of scientific instruments-are not tied to any particular broad perspective, and thus if they are perspectival, they are so in a much broader sense. Second, it is established that when broad perspectives change, objectivity standards do not change concomitantly. And third, by drawing on two brief case studies-the history of the cloud chamber (Galison 1997) and of stellar classifications (Hoffeit 1991) -it is established that scientific instruments and their outputs can cut across both broad perspectives and changing objectivity standards. Thus, as long as shifts in objectivity standards are not abrupt and discontinuous and do not correspond to shifts in broad perspectives, knowledge derived from scientific instruments can be objective despite changes in broad perspectives.

The second strategy is developed around narrow perspectives to respond to the theory-ladeness challenge in relation to scientific instruments. I show that narrow

\footnotetext{
${ }^{2}$ See Laudan (1990) for a comprehensive discussion of both problems, and New Experimentalists, such as Hacking (1983), Franklin (1990, 1986, 2015), Mayo (1996), and Franklin and Perovic (2019) on epistemic strategies to overcome the theory-ladeness of experiments, as well as other skeptical challenges.

${ }^{3}$ See Creţu (2020c) for an accessible overview.

${ }^{4}$ For more general problems with perspectivism, see Chakravartty (2010), Chirimuuta (2016), and Morrison (2011). For a sustained defence of perspectivism, see Massimi (2012, 2018a, 2018b, 2018c, 2018d).

${ }^{5}$ Broad perspectives are discussed by Massimi (2018c) and narrow perspectives are discussed, in different ways, by Massimi (2012) and Creţu (2020a). The current article builds on and extends these earlier analyses in a systematic way and applies them in a new context.
} 
perspectives, which constitute the working stances of scientists, are restricted neither to one theory nor just to theory. As such, narrow perspectives contain within them cross-perspectival justificatory tools to render instruments and their data valid and objective.

This paper will be of value to philosophers, scientists, and historians who take scientific instruments to deliver knowledge about the world. Since the challenges posed by perspectivalist claims threaten to undermine the process of scientific knowledge production, any disruption to the process of using instruments to obtain scientific knowledge is worth investigating in its own right.

The article is structured as follows: section 2 examines notions of "perspective" and clarifies Giere's account. In section 3, the problem of conceptual relativism is analyzed in terms of the instrumental incommensurability between broad perspectives. Thus reframed, the problem can be resolved via reference to two novel responses, the first "objectivity-led" and the second "instrument-led." In section 4, the problem of "instrumental theory-ladeness" is separately resolved with reference to individual scientists' narrow perspectives. It is ultimately demonstrated that scientific instruments can be understood as perspectival in two distinct senses, each leading to distinct challenges for which systematic responses are provided.

\section{Perspectives clarified}

To determine what, if any, the import of scientific perspectivism is in relation to scientific instruments and whether, in this context, it brings about either new epistemic challenges or new solutions to such challenges, two steps are necessary. First, it is necessary to understand what kind of "perspectives" are relevant to instruments. Second, it is necessary to understand in what ways scientific instruments depend on relevant perspectives and what epistemic consequences may result from such dependence.

\section{I Intuitive perspectives}

Consider, to begin with, two of the most common meanings associated with perspectives: the private, personal point of view and the human point of view. Regarding the first, we interact with the world from our point of view and not from another's point of view, and in a deep sense, we cannot entirely escape our point of view. Regarding the second, we cannot know things from a nonhuman point of view, and thus, whatever we do know from the human point of view is all we can know. Although it is important to acknowledge these limitations, they are nonetheless insufficient insights for a full analysis of the perspectivity of scientific instruments.

The perspectivity of instruments could be further taken to mean something about the way humans "see" with and through instruments (Giere 1999, 2000, 2006). Not all humans are equipped with the same visual system. Although most humans have trichromat vision that enables them to see the full spectrum of colors, some humans are color-blind. Most frequently, color-blind humans cannot distinguish red and green, and in some cases, complete color-blindness precludes the experience of colors. If instruments are perspectival because the results they yield depend on the visual system that manipulates them, their perspectivity might not be trivially 
true. In and of itself, the claim that different visual systems can yield different kinds of knowledge about the world is not epistemically problematic. Suppose Cat and Pat are using exactly the same microscope to look at an insect wing. If Cat cannot see any colors and Pat can, they will naturally see the insect wing differently, Cat from a monochromatic perspective, Pat from a colorful perspective. Their perception of the insect wing will be inherently different, one without color information, the other with color information. Thus, because of their different visual systems, Cat's and Pat's investigations with the same instrument can yield different kinds of knowledge about the world. What this example suggests, however, is that the perspectivity under discussion has nothing to do with the instrument in question. Cat and Pat would see the insect wing differently with or without the instrument because it is their visual system that constitutes the relevant perspective, and not anything to do with the instrument itself.

\subsection{Scientific perspectives}

We may further assume that what makes instruments perspectival are the following perspectival features: (i) "they respond only to a limited range of aspects of their environment" (Giere 2006, 41); (ii) "even for those aspects of the world to which they do respond, the response is limited" (41); and (iii) they "have some limitations on their ability to discriminate among inputs that are theoretically distinct" (42). Evans (2020) recently discussed the first two features in his account of "perspectival objectivity," and points of agreement or disagreement will be highlighted below.

The claim that scientific instruments are perspectival because they have a limited range should hardly be controversial. It is simply to say that a scientific instrument will deliver knowledge of only some aspects of the world but not others. This is nevertheless self-evident: every observation from the vantage point of a particular instrument is limited to the range of inputs detectable by the relevant instrument. For example, a telescope is useless for detecting positrons simply because the energy scale of the positrons is not detectable by telescopes. As Evans (2020) points out, as humans, we are similarly "limited" in that "we are sensitive only to a certain set of variables, namely ones that can be detected by sight, sound, touch, and taste" (5). This insight, whilst useful, does not seem to fully capture whatever it is that makes instruments perspectival.

The second respect in which instruments can be deemed perspectival is through their limited response to limited inputs. To use Giere's example, "[a] camera responds only to radiation to which its film or more recently, its digital sensors are attuned" (42). Because "[e]very instrument interacts with the world only from its own particular perspective" (Giere 2000,10), and "part of the perspective of any instrument is determined by its built in margin of error" (11), instruments can only yield partial, perspectival knowledge. This, as Evans (2020) emphasizes, "is a trivial observation, in the sense that we cannot model undetectable properties or behaviour of a system in terms of undetectable variables" (5). According to Evans, this observation discloses, nevertheless, something about the limitations of the perspective through which we can interact with and model the world. Although Evans is right to emphasize that this point exposes the role of our "idiosyncratic capabilities to interact with, and model, 
reality" (5), we can only interpret this claim as circumscribing the perspective of the instrument from, evidently, our own vantage point. ${ }^{6}$

The third respect in which instruments are judged to be perspectival consists in their inability to discriminate between theoretically distinct inputs. Prima facie, this may strike one as a misplaced charge because instruments do not have agency, and thus they are not meant to distinguish between theoretically distinct inputs. But because Giere insists that "claims about what is observed cannot be detached from the means of observation" (Giere 2000, 48) and that "[o]ne cannot detach the description of the image from the perspective from which it was produced" (56), the indiscriminateness of instruments is due to their theory-ladeness. Realists, relativists, positivists, and pragmatists alike have long agreed that "theories are involved in the construction and interpretation of instruments" (Laudan 1990, 47) and that "theoretical assumptions go into determining the boundary conditions supposed to apply to any situation under scrutiny" (47). One may nevertheless insist that there is something deeply perspectival about cases of circular theory-ladeness in which the theory of the instrument and the theory of the phenomena are mutually reinforcing. This is a legitimate worry examined in section 4.

The most promising interpretation of the perspectivity of instruments is as highlighting the dependence of instruments on different kinds of perspectives. The personal, the human, and the visual perspectives were diagnosed as having little bearing on scientific instruments, whereas additional notions proposed by Giere require further development. In particular, understanding perspectives as "a way of constructing scientific models" (Giere 1999, 79) or a particular culture (Giere 2013) makes for a useful, yet overly broad, dependence of instruments on such perspectives. Similarly, the standpoint of a scientific community or the observational standpoint of an observer or of an instrument (Giere 2006) makes for a rather coarsegrained dependence. In the remainder of this article, it will be shown that finergrained notions, that is, broad perspectives (Massimi 2018a) and narrow perspectives (Massimi 2012; Creţu 2020a), can be exploited to deliver novel solutions to both conceptual relativism and theory-ladeness.

\section{Broad perspectives}

This section explores the potential benefits of thinking of perspectives as historically and intellectually situated scientific frameworks typical of a scientific community, along the lines of Massimi (2018a). According to Massimi (2018a) such perspectives, let us call them broad perspectives, encompass "(i) the body of scientific knowledge claims advanced by the scientific community at the time; (ii) the experimental, theoretical, and technological resources available to the scientific community at the time to reliably make those scientific knowledge claims; and (iii) second-order (methodological-epistemic) claims that can justify the scientific knowledge claims so advanced" (343). Thus defined, broad perspectives are better thought of not as a specific theory but as research traditions. ${ }^{7}$ Like research traditions, broad perspectives sponsor a variety of norms, background assumptions, "narrow perspectives,"

\footnotetext{
${ }^{6}$ Baker (2020) offers a similar diagnosis.

${ }^{7}$ This is a gloss on Massimi (2018a), who acknowledges but does not clarify dissimilarities between historical practices and contemporary modeling practices.
} 
and theories alike, as well as theories about instruments, their operation, and their interpretation. ${ }^{8}$

However, although each such perspective can produce, through its instruments and their outputs, its own conceptualization of the world, any such conceptualization can become essentially nontransferable across broad perspectives, leading to a form of incommensurability-let us call it instrumental incommensurability-that can lead, in turn, to conceptual relativism. In what follows, two novel responses to instrumental incommensurability are offered, an "objectivity-led response" and an "instrument-led response." But before we turn to these two responses, it will prove instructive to understand how the challenge arises.

\section{I Instrumental incommensurability}

Broad perspectives, understood as research traditions, articulate, through the elements they sponsor, particular conceptualizations of the world. For example, broad perspectives sponsor objectivity standards, such as "truth-to-nature" (standardized types), "mechanical objectivity" (proceduralized automation), "structural objectivity" (communicable invariance), or "trained judgment" (skilled interpretation). ${ }^{9}$ Briefly, the first objectivity standard refers to the practice of the seventeenth- and eighteenth-century natural philosophers to whom the variability of individuals in nature possessed too great a danger for subjective distortion. For them, objectivity consisted in selecting and perfecting an archetype that could truly stand for the whole class. This practice declined in popularity around the 1830 s (although it did not vanish) with the rise of the second standard of objectivity. The era of mechanical objectivity, extending to the 1920s, sought to recuperate the variability of the individual through mechanized procedures that would suppress the predecessors' predilection to prettify. Alongside this practice, although in reaction to it, structural objectivity was developed around the 1880s. This was a standard that sought to extract only those invariable, structural features that could be communicable to one and all. In reaction to this sanitized objectivity standard and as a result of increasingly complex instrumentation, the 1920-1930s saw the emergence of a new standard of objectivity, trained judgment, which relied on the hard-won skill of the scientist to detect patterns and interpret complex families of phenomena.

Each of these objectivity standards was deployed in scientific practice, and specifically in the use of scientific instruments, in order to suppress problematic subjectivity in its many guises, from the predilection to "prettify, idealize" and distort or project to the tendency to "regularize observations to fit theoretical expectations" (Daston and Galison 2007, 34). However, if such objectivity standards turn out to be perspectival (because they are embedded within a particular broad perspective), this might suggest that instruments and their outputs might themselves be found to be perspectival. Were this scenario to prevail, one might rightly conclude that instruments cannot yield objective knowledge because they are essentially laden to a particular point of view (albeit a broad one in this case). And thus, broad perspectives, like

\footnotetext{
${ }^{8}$ See Laudan (1977) on research traditions and theories, and see Creţu (2020a, 2020b) on research traditions, perspectives, and theories.

${ }^{9}$ A broad perspective sponsors or endorses objectivity standards, but it does not necessarily create them.
} 
their Kuhnian predecessors, could lead to pervasive Kuhnian "paradigm-ladeness" and further problems thereto.

One particularly thorny problem is conceptual relativism, typically brought about by some form of incommensurability. Conceptual relativism is an old problem for scientific knowledge (cf. Laudan 1990), although not usually concerned with scientific instruments. Let us nevertheless assume that conceptual relativism can be brought about by some form of discontinuity or incommensurability at the level of the instruments themselves-let us call this instrumental incommensurability. If and when broad perspectives change and such change leads to either knowledge of instrumentbuilding and operation or knowledge delivered by instruments becoming essentially nontransferable from one broad perspective to the next, the instruments can be said to be "internal" to that perspective (Giere 2006, 49). By specifying in what ways instruments can be said to be internal to a broad perspective and what challenges may arise, we can then show how the relevant challenges can be resolved.

In principle, there are at least three different ways in which instruments can be said to remain internal to a broad perspective and become essentially nontransferable:

i. When there is physical nontransferability of instruments and their outputs across broad perspectives

ii. When nontransferability of technical knowledge occurs, understood as either practical or as theoretical knowledge of instrument-building and operation

iii. When conceptual nontransferability of instruments and their outputs occurs

In practice, it is difficult to find examples of radical discontinuity in the history of instruments for the simple reason that it is difficult to find things that were lost. Even the famous astrolabe of the Hellenistic world of Alexandria, despite being materially lost to later astronomers, resurfaced in medieval Islam through texts and practices (see Hayton 2012 and King 1992). Relatedly, feeding scepticism about radical discontinuity, even musical instruments confined to the history books, such as the Epigonion and the Barbiton of Ancient Greece, have been recently recreated (Avanzo et al. 2010). Nonetheless, it will be instructive to examine the different ways in which instrumental discontinuity can occur in order to identify the attending challenges.

Broad perspectives are extended in time, and thus the physical nontransferability of instruments, as well as of their physical outputs, is unfortunately unavoidable. Instruments may become irreproducible on certain timescales because of a lack of material resources or can become obsolete when more efficient alternatives are developed (i.e., less costly, more sustainable, etc.). Instruments are also likely to deteriorate, can be moved, can fall into disrepair, or might fall prey to accidents and natural disasters (cf. Schaffer 2011). All these possibilities can make instruments, as well as their outputs, physically nontransferable across broad perspectives. This kind of nontransferability, even if seen as some form of broad perspectivity, would nevertheless be entirely accidentally located within a broad perspective. For accidental occurrences that may lead to the destruction of instruments to coincide with the cutoff point of the transition from one broad perspective to another is not only an entirely contingent matter, but it is also exceedingly unlikely. If this were 
nonetheless to occur, it could lead to a form of (radical) conceptual relativism brought about, inter alia, by instrumental incommensurability between broad perspectives. To be precise, because different instrumental perspectives can be said to construct the world differently, any discontinuity between such perspectives may preclude any commonly established facts between the relevant perspectives, thus leading to conceptual relativism. Although this is a serious problem for the progress of science, conceptual relativism is neither a distinctively perspectival challenge (but an old Kuhnian challenge), nor is it a challenge specific to, or restricted to, scientific instruments. ${ }^{10}$

Unlike the physical nontransferability of instruments, the technical nontransferability of instruments is more likely to occur. ${ }^{11}$ Yet, like the physical nontransferability of instruments, technical nontransferability amounts to the same type of Kuhnian challenge because nontransferability precludes the possibility of commonly established facts (because the facts can no longer be produced, recognized, etc.). Thus, although one can rightly take the technical nontransferability of instruments as a form of perspectivism, it remains to be shown that this kind of perspectivism is distinctively different from Kuhnian paradigm-ladeness, which can be said to lead to conceptual relativism via instrumental incommensurability.

Finally, the conceptual nontransferability of instruments and their outputs across broad perspectives constitutes an equally serious problem as their technical nontransferability. If instruments and their outputs are conceptually laden to broad perspectives, then each broad perspective produces, through its instruments and their outputs, its own conceptualization of the world. To put it differently, the nontransferability of instruments and their outputs across broad perspectives means that all knowledge yielded by instruments can only be knowledge from within a perspective. This amounts to a type of perspectivity akin to conceptual relativism. Insofar as perspectivity amounts to conceptual relativism, there is, once more, no novel epistemic import of perspectivity. However, although conceptual relativism is not a distinctively perspectival challenge, two distinctively perspectival responses are available in relation to instruments, as will be argued in the remainder of this section.

First, an important clarification is in order. We assumed that objectivity standards are perspectival, that is, that they are specific to a broad perspective. ${ }^{12}$ We further assumed that if broad perspectives are regarded as insular and disjointed, then changes in broad perspectives automatically lead to wholesale changes, including changes in objectivity standards, which can bring about conceptual relativism. Yet there are three strong reasons to resist our previous assumptions:

i. First, the scholarship on the history of objectivity suggests that objectivity standards are not tied to any particular broad perspective (and thus if they are perspectival, they are so in a much broader sense).

\footnotetext{
${ }^{10}$ For an excellent discussion of the intricacies of relativism and different solutions, see Laudan (1990), for an overview see Baghramian and Carter (2019).

${ }^{11}$ See Hicks (2017) on technical discontinuity and Galison (1997) on technical continuity.

${ }^{12}$ This assumption would be warranted within Giere's (2006) perspectivism.
} 
ii. Second, when broad perspectives change, objectivity standards do not change concomitantly.

iii. Third, and most importantly, scientific instruments and their outputs typically cut across both broad perspectives and changing objectivity standards.

The first two reasons can be combined to mount a novel "objectivity-led" response to the instrumental incommensurability challenge, developed in section 3.2, whereas the third reason guides a novel "instrument-led" response, developed in section 3.3.

\subsection{Objectivity-led response}

In this section, it is shown that objectivity standards are not inherently tied to any particular broad perspective, nor do they necessarily change concomitantly with any broad perspective. On the contrary, it is shown that older standards can survive alongside succeeding predominant standards, even past the predominance of the succeeding standard. For example, as Daston and Galison indicate, "truth-to-nature" survived alongside not only "mechanical objectivity" but also alongside "structural objectivity" and "trained judgment." Thus, if, as Daston and Galison (2007) suggest, objectivity standards changed first and foremost in response to particular subjectivity threats, such as prettifying, idealizing, distorting, projecting, or regularizing observations to fit theoretical expectations, we can reasonably assume that each objectivity standard "strove" away from perspectivity to apersepctivity. Hence, even if objectivity standards are perceived as in some way perspectival, continuity in objectivity standards, over and above changes in broad perspectives, should suffice to avert perspectivism-cum-conceptual relativism in relation to scientific instruments.

Daston and Galison (2007) identify four types of objectivity, each predominant in different periods, although each is relevant to other notions of objectivity over considerably longer periods of time past their predominance. Truth-to-nature, the first standard of objectivity identified by Daston and Galison (2007), was predominant in the seventeenth and eighteenth centuries. It consisted in identifying idealized types - arrived at through reasoning and selection. Idealized types could not be found in nature but were taken to be "truer" to nature than any unruly token. Tokens had fleeting features that had to be brought within an objective type through selection, synthesis, and idealization. To the seventeenth- and eighteenth-century naturalists, "unreasoned observations" were considered subjective, whereas the "idea in the observation" and not the observation itself was considered objective, true-to-nature.

The truth-to-nature objectivity standard changed around 1830, giving way to mechanical objectivity, which regulated scientific practice for nearly a century. New means of mechanization and automation promised deliverances of instruments, such as photographic images, uncontaminated by the dangerous distortions of reasoned images. When automation was not possible, exceedingly proceduralized means of recording nature without distortion or interpretation were developed, which often involved "humans acting as will-less machines" (Daston and Galison 2007, 120). Yet, no automated instruments could offer "pure," unadulterated access to nature. For example, depth of field or color could not be precisely recorded by automatic means, leading to accuracy being traded off for mechanical reproduction. The preponderance of such trade-offs, despite automation, left an ineliminable 
human element that the succeeding image of objectivity, structural objectivity, sought to suppress.

Structural objectivity emerged in the 1880s, coexisted with mechanical objectivity till at least the 1920s, and is currently still embraced by scientists and philosophers with a structuralist bent. Structural objectivity, which must be "communicable to all" and according to which the "private mental world of individual subjectivity" (Daston and Galison 2007, 254) has no place in the epistemology of nature, is not always applicable to instruments, except maybe for logic devices such as counters, spark chambers, and wire chambers (see Galison [1997] for details on logic devices). This is because structural objectivity is primarily concerned with "enduring structural relationships that survived mathematical transformations, scientific revolutions, shifts of linguistic perspective, cultural diversity, psychological evolution, the vagaries of history, and the quirks of individual physiology" (Daston and Galison 2007, 259) and not, strictly speaking, with the deliverances of instruments. Although not pertaining directly to instruments, structural objectivity is worth bringing into the present discussion because it was concurrent with mechanical objectivity, and it neither occurred with nor shifted concomitantly to broad perspectives.

Structural objectivity proved insufficiently versatile for understanding complex families of phenomena, and thus a new form of objectivity, trained judgment, became predominant in the mid- to late twentieth century. Trained judgment was needed to "synthesise, highlight, and grasp relationships" and to "smooth, refine, or classify images" (Daston and Galison 2007, 314). In stark contrast with both mechanical objectivity and structural objectivity, which sought to extirpate individual judgment, trained judgment relied on an individual's ability to "read, to interpret, to draw salient, significant structures from the morass of uninteresting artifact and background" (ibid., 328). Trained judgment relied on the human ability to "seize pattern" and to obtain "knowledge at a glance," skills that were "acquired through a sophisticated apprenticeship" (ibid., 331). Interpretation, previously conceived as epistemically problematic and as stunting the effort to "get at the world," was now conceived as necessary to interpret the ever-more-complex images produced by sophisticated instruments.

Thus, as the history of objectivity distinctly indicates, there is no abrupt shift from one standard of objectivity to the next. In fact, two or more standards of objectivity survive alongside one another, whereas the transition from one standard to another is clearly traceable. Unlike the traditional Kuhnian narrative of wholesale paradigm changes, shifts in objectivity standards are not wholesale. Not only does each type of objectivity safeguard against specific types of subjectivity and thus screens off epistemic threats such as "drowning in details, of burking a fact to support a theory, of being straitjacketed by mechanical procedures" (ibid., 377), all of which are "genuine dangers to knowledge" (ibid., 377), but each objectivity standard also builds on and reacts to earlier specific threats to knowledge. Therefore, as Daston and Galison (2007) suggest, it "is a misconception, albeit an entrenched one, that historicism and relativism stride hand in hand" (ibid., 376).

If one accepts Daston and Galison's (2007) history of objectivity, another important observation becomes salient: objectivity shifts do not correspond to shifts in broad perspectives. For example, Lorentzian ether theory and special relativity can both be said to be governed by structural objectivity, despite being different broad 
perspectives (for the history of special relativity and Lorentzian ether theory, see Brown [2005]). Similarly, trained judgment governed cloud-chamber experiments both prior to and after the crystallization of relativistic quantum mechanics (Creţu 2020a; Roqué 1997). Scientists, in such cases, successfully navigated not only shifts in broad perspectives but also later shifts in objectivity standards (Galison 1997). What the history of objectivity demonstrates is that conceptual relativism can be avoided with respect to scientific instruments as long as shifts in objectivity (i) are not abrupt and discontinuous and (ii) do not correspond to shifts in broad perspectives. Thus, knowledge derived from scientific instruments can be objective despite changes in broad perspectives.

What if broad perspectives are broader $?^{13}$ That is, what if the objectivity standards themselves define the perspectives, and when they shift, the instruments and their deliverances also shift? To be clear, the critic may insist that the objectivity-led response leaves open the possibility that instrumental incommensurability occurs when objectivity standards shift. Drawing on Galison (1997) and Hacking (1983), who have presented detailed case studies involving instrument-led continuity despite changes in theory, a novel answer that demonstrates instrument-led continuity despite changes in objectivity standards is offered in the next section.

\subsection{Instrument-led response}

The second response to instrumental incommensurability resides in the fact that instruments and their outputs typically do not change when objectivity standards change. To be clear, the claim is that when objectivity standards shift, scientists who adhere to the new predominant standard can nevertheless use instruments designed according to previous objectivity standards. Importantly, the outcomes of such instruments are not typically contested either. This is not to say that in some cases, instruments and their data may not lose their original significance or the data may not be interpreted differently. This can, of course, occur, but typically, the data stay the same (cf. Ackerman 1985, 33). To illustrate this claim, let us consider two brief examples.

The first example, drawn from physics, is primed to illustrate the fact that instruments and their deliverances can be markedly cross-perspectival, even when perspectives are defined by the objectivity standards themselves. That is, despite being built or used to deliver data under the auspices of one objectivity standard, the instrument and the data produced often successfully survive shifts in objectivity standards. The cloud chamber constitutes one example of an instrument spanning a lengthy and versatile career through shifting objectivity standards. The cloud chamber is undoubtedly one of the most important instruments of the twentieth century. ${ }^{14}$ It played an important role in many Nobel Prizes in Physics, and it gave rise to the tradition of "golden events"-that is, the tradition of making visible and capturing on film the interactions of subatomic phenomena. ${ }^{15}$

${ }^{13}$ Thanks to Nicos Stylianou for very useful discussions on this issue and for pushing me to clarify this point.

${ }^{14}$ The historical details are drawn from Galison (1997), Das Gupta and Ghosh (1946), and Blackett (1960).

${ }^{15}$ See Staley (1999). 
The cloud chamber was invented by C. T. R. Wilson in 1911 under the sponsorship of mechanical objectivity. While pursuing research in atmospheric phenomena, Wilson recorded the first golden event, of an alpha ray, in 1911. The cloud chamber, although developed by Wilson for the study of atmospheric phenomena, was soon appropriated by the Cavendish physicists to study subatomic phenomena. After a series of tweaks and improvements, the cloud chamber gave rise to further golden events, such as the photograph of the positron published by Carl D. Anderson in 1932 and the joint discovery of the muon by Anderson and Seth H. Neddermeyer in 1936. The tradition of golden events, inaugurated by C. T. R. Wilson under the auspices of mechanical objectivity, gathered momentum under the sponsorship of trained judgment with Anderson's discovery of the positron, and it is still very much alive almost a century later and in spite of various changes in objectivity standards. Importantly, neither C. T. R. Wilson's photograph of alpha rays nor Carl Anderson's photograph of the positron has lost its significance, nor has Wilson's cloud chamber or Anderson's cognate apparatus been called into question. These photographs were produced from the vantage point of perspectives governed by objectivity standards different from those that originally governed the invention of the cloud chamber and were embedded within successive broad perspectives governed by yet different objectivity standards. Thus, what we can learn from the history of the cloud chamber is that changes in objectivity standards have not transformed or denied the significance of either the cloud chamber itself or its capacity to produce golden events. To put it differently, its ladeness to different norms of objectivity did not bring about conceptual relativism via instrumental incommensurability. On the contrary, it is clear that the use and importance of the cloud chamber cut across both broad perspectives and changing objectivity standards, and the instrument is thus markedly cross-perspectival.

The second example, drawn from astrophysics, focuses on the data rather than the instruments per se. It shows that even when certain instruments become obsolete, their deliverances retain their original authority despite shifts in objectivity standards. The example concerns the photographic plates of the spectra of stars that became available toward the end of the nineteenth century with the invention of the spectroscope ${ }^{16}$ By attaching a prism or a slit to a telescope to separate the rays of starlight by their wavelengths, their unique spectra or absorption lines can be recorded, and their brightness can thus be measured. The first successful attempt to photograph the spectra of stars belongs to Henry Draper, who photographed the spectrum of Vega in 1872, with similar research also being conducted by William and Margaret Huggins.

Henry Draper's photographic plates were analyzed and measured by Edward C. Pickering and the "human computers" working alongside him at the Harvard Observatory, Williamina Fleming, Antonia Maury, and Annie Jump Cannon. ${ }^{17}$ This analysis constituted the catalyst for the creation of the Henry Draper Memorial, which gave rise to the Draper Catalogue of Stellar Spectra, first

\footnotetext{
${ }^{16}$ The historical details are drawn from Hoffeit (1991).

${ }^{17}$ Human computers refers to scientific workers who performed calculations or reduced and analyzed data before the advent of digital computers; for details, see Light (1999).
} 
published in 1890 by Pickering and Williamina Fleming, with subsequent installments in 1897 from Antonia Maury and in 1901 from Annie Jump Cannon. The Draper Catalogue of Stellar Spectra constitutes the first modern classification of stars and is the forerunner of both the Henry Draper Catalogue (published between 1918 and 1924 by Annie Jump Cannon) ${ }^{18}$ and Morgan, Keenan, and Kellman's 1943 Atlas of Stellar Spectra.

Importantly, for our purposes, the Henry Draper Catalogue was designed according to the canons of mechanical objectivity, which were quintessentially empirically driven and implied "automation" and abstention from theory, whereas Morgan, Keenan, and Kellman's Atlas of Stellar Spectra relied on trained judgment, skill, and interpretation. Yet despite the use of mechanical objectivity, the results of the Henry Draper Catalogue were not only fully understood and recognized by their successor, but the spectrographic photographs on which they were based preserved, in bulk, their significance. Although Morgan, Keenan, and Kellman used different instruments, published new photographs, and operated with a distinct objectivity standard that relied on their skill and interpretation (i.e., trained judgment), they nevertheless cite the Draper Catalogue as the direct forerunner of their own classification (cf. Morgan et al. 1943; see also Daston and Galison 2007). The spectrographic photographs that constitute the basis of the Henry Draper Catalogue of Stellar Spectra can thus be said to cut across shifting objectivity standards. What this example suggests, then, is that changes in objectivity standards did not lead to the conceptual relativism brought about by instrumental incommensurability.

Two examples cannot conclusively show that instrumental incommensurability cannot occur or that it does not lead to conceptual relativism when objectivity standards shift. Reflecting on these examples suggests, nevertheless, that conceptual relativism can be avoided when instruments and their deliverances survive shifts in objectivity standards. Moreover, conceptual relativism via instrumental incommensurability can also be avoided when shifts in broad perspectives do not correspond to shifts in objectivity standards. Thus, instead of constituting a novel epistemic challenge, the dependence of instruments on broad perspectives, when closely analyzed, provides instead the means to mount a novel response to the old epistemic challenge of conceptual relativism.

To sum up, it was shown that not only are objectivity standards and broad perspectives not concomitantly shifting, but instruments and their deliverances can also cut across both broad perspectives and objectivity standards. So although objectivity standards and instruments are in some respects perspectival, by being historically and intellectually situated both within and outside broad perspectives, they are equally cross-perspectival. Thus, the scientific instruments themselves and the objectivity standards governing them equally constitute tools to block the conceptual relativism brought about by instrumental incommensurability.

${ }^{18}$ Two other extensions, the Henry Draper Extension, published between 1925 and 1936, and the Henry Draper Extension Charts, first published in 1937, were assembled by Annie Jump Cannon and Margaret Mayall. 


\section{Narrow perspectives}

This section examines the nature of the dependence of instruments on "narrow perspectives," the resulting problem(s), and a potential solution. Narrow perspectives, unlike broad perspectives, have a significantly restricted scope. However, they are not restricted to a single theory or model. Narrow perspectives are distinctively perspectives or points of view, unrestricted to any particular theory (Creţu 2020a) or just to theory (Massimi 2012). Narrow perspectives can be understood as "sophisticated theoretical framework[s] that encompasses the set of theoretical interests and background theoretical knowledge (principles and assumptions equally) that a researcher or group of researchers can be said to hold at any given time" (Creţu 2020a, 29). Or a narrow perspective can be constituted by a scientist's epistemic perspective, which includes beliefs about the phenomena under investigation, the correct functioning of instruments, and the validation of their outputs, but also more general beliefs about their "perceptual system, cognitive faculties, measurement devices, and their reliability as sources of belief" (Massimi 2012, 41). Either notion of perspective characterizes the working stance of a scientist, who, in one capacity or another, comes to have a bearing on scientific instruments, directly by constructing or using them or indirectly by affecting the data. Because the use of scientific instruments can thus become laden to a scientist's (narrow) perspective, elucidating the nature of the ensuing ladeness will prove highly instructive.

\section{I Instrumental theory-ladeness}

This section offers an explication of the relationship between instruments and theories to show that reliance on theory is, for the most part, invaluable to using instruments and interpreting their data. However, complications arise once scientists' (narrow) perspectives are taken into account, leading to "instrumental theory-ladeness." This variation of the theory-ladeness challenge is explained in this section, and a solution is proposed in the final section.

Scientific instruments may become laden to a scientist's viewpoint in a variety of ways. Theory-ladeness can occur (i) in cases where the theory governing the instrument and the theory of the phenomena are one and the same; (ii) in cases where there exists an overattachment to the theory that clashes with the recalcitrant data produced by an instrument; or (iii) as a result of practical problems, such as "experimental design, failure to interpret observations correctly, possible experimenter bias, and difficulties in data acquisition" (Franklin 2015, 155).

In the first case, theory-ladeness is avoided by ensuring that the theory governing the instrument and the theory of the phenomena are different. As Franklin argues, "no obvious problems arise for the testing of the theory of the phenomena" in such cases (Franklin 2015, 439n8). And even in cases in which the theory governing the instrument and the theory of the phenomena partially overlap or overlap to a large extent, successful strategies for overcoming vicious circularity have already been suggested in the literature. For example, as early as three decades ago, Franklin et al. (1989) discussed the possibility of using an instrument whose operation depends on the same hypothesis as that of the phenomena under test and suggested that in such cases, the calibration of the instrument should suffice to mitigate the threat of vicious theory-ladeness. The instrument under test would be independently 
calibrated against an already-validated instrument by measuring a different phenomenon whose theory overlaps with neither instrument. Later, Chalmers (2003) offered a detailed case study of the electron microscope, showing that in spite of a deep theoryladeness, instruments could nevertheless be used to collect data about phenomena, even in cases where the theory of the phenomena was involved in the use of the instrument. As Chalmers notes, "[t]he interdependence of theory and data ... can, in appropriate circumstances, be exploited in a way that confounds rather than aids the sceptic" (494). Recently, Beauchemin (2017) showed that in the case of conglomerate instruments such as the ATLAS detector at the Large Hadron Collider (LHC), theory input may be essential to confer epistemic value to certain measurements. ${ }^{19}$ In fact, "theory-ladeness of measurement is necessary for [measurements] to constitute observations" (309), and often, progress in high-energy physics can only be made by mutually adjusting theory and experiment. The same point, although for the case of astrophysics and cosmology, was also made by Bondi (1955), who notes that "[t]o derive any significant astronomical result from the blackening of a photographic plate or the simple reading of a meter a tremendous amount of intervening work has to be done. Corrections may have to be applied, calculations and reductions may have to be carried out, and above all interpretations requiring a great deal of theoretical background may have to be made" (157).

The main point to note in connection to these examples is that once we examine more closely the relevant theory-ladeness of an instrument, it becomes clear that the import of theory is, for the most part, invaluable and not particularly problematic. To be clear, the claim is that regardless of how multiply perspectival the process of using instruments can become-from using a thermometer to using an electron microscope or a spectrograph to conglomerate instruments such as the ATLAS detector at the LHC-there are epistemic strategies for avoiding vicious theory-ladeness. Insofar as such epistemic strategies can be deployed, instruments remain an objective source of knowledge about the world.

Concerning the overattachment to the theory that clashes with the recalcitrant data produced by an instrument, a novel complication arises in relation to scientists' (narrow) perspectives. The idea is that scientists' (narrow) perspectives will typically play an important role in accepting new data or in discarding it. For example, if the data are unexpected, "scientists with different perspectives may respond differentially to the same empirical knowledge, ... impeding [the] authentication" of some hitherto unknown phenomena (Creţu 2020a, 2) and leading to disagreement. In such cases, regardless of the route taken to resolve the disagreement, scientists' (narrow) perspectives inadvertently affect the use of the instruments and the interpretation of their outputs.

Scientific instruments are not only dependent on theory or just one theory, but they are also dependent on laws and background conditions, sometimes weather conditions; they are embedded within experiments; and experiments themselves can be theory-laden in a variety of ways, as was recently argued, in different ways, by Karaca (2013) and Schindler (2013). ${ }^{20}$ When a scientist responds to recalcitrant data, they may be objecting to any of the several elements involved in the use of

\footnotetext{
${ }^{19}$ Thanks to Antonis Antoniou for pointing me to Beauchemin's work.

${ }^{20}$ See also Franklin and Perovic (2019).
} 
an instrument and the production of the data. Importantly, in accepting or discarding the data, the scientists who interpret the data may call into question the instruments themselves, despite the lack of any obvious practical problems, such as "experimental design, failure to interpret observations correctly, possible experimenter bias, and difficulties in data acquisition" (Franklin 2015, 155). To understand why a scientist may accept or reject specific data, we need to understand their narrow perspectives. That is, we need to understand in which ways their assumptions or interests interact with the instruments and the data. This complex relationship between instruments and the production of data and narrow perspectives adds very specific extra layers of theory-ladeness and constitutes a distinct variation on previously acknowledged forms of theory-ladeness. Let us therefore call it instrumental theory-ladeness and consider a possible response to it in the final section.

\subsection{Perspectivity-led response}

The claim that instruments are perspectival derives in large measure from the observation that in the construction and use of instruments and the interpretation of their data, there are multiple layers of theory involved. This is not particularly surprising because nearly no scientific instrument, if any, ever delivers unadulterated access to incontrovertible facts (Bondi 1955). Some philosophers, such as Giere (2006), have nonetheless taken this perspectivity of instruments to mean that they cannot deliver objective empirical knowledge. However, the fact that theory-ladeness can lead to vitiated results does not mean that it inevitably does so. In this section, a more positive interpretation of this challenge is provided by means of explicating the heuristic role of narrow perspectives.

In the first instance, it will be useful to clarify what kind of perspectivity can be involved in the theory-ladeness of instruments, specifically in relation to a scientist's (narrow) perspective and when and why the resulting perspectivity might be problematic. In talking about a scientist's perspective, we can, and we often do, distinguish between, on the one hand, their prejudices, idiosyncrasies, and other subjective elements of their perceptual systems and cognitive faculties and, on the other hand, their background knowledge and theoretical interests. The former is their subjective perspective; the latter is their (narrow) epistemic perspective. The subjective perspective of a scientist cannot be entirely eliminated from the scientific life, but there are many variegated levels of correcting for the ills of subjectivity. The adoption of a standard of objectivity is one way through which subjectivity can be kept in check (as shown in section 3). Another way to correct for subjectivity is to identify and analyze the scientist's narrow perspective and to check their methods and justifications in relation to instruments.

Narrow perspectives can encompass both entrenched background assumptions and less entrenched ones pertaining to particular problems salient to working scientists (Creţu 2020a, 32). Furthermore, narrow perspectives enable scientists to "self-reflect on [their] beliefs, on the sources of [their] beliefs, the way beliefs cohere with one another, no less than the way in which they, individually and jointly, are anchored to the empirical ground via reliable methods" (Massimi 2012, 49). By examining a scientist's (narrow) perspective, we gain access to their methods, reasons, and justifications for building a particular instrument, using a particular 
instrument, or interpreting the results of a particular instrument in a specific way. Such knowledge allows us to ascertain what epistemic strategies have been used to avert the dangers of vicious theory-ladeness or the ills of subjectivity, such as making observations fit theoretical expectations.

We usually find that strategies such as those identified by the New Experimentalists (see footnote 2) have been applied to ensure that the results of an experiment are accurate and reliable and are to be trusted with a high degree of certainty, even though they cannot ever be absolutely certain. Such strategies, as identified and discussed by Franklin (1989), where some pertain directly to instruments while others pertain more generally to experiments, include the following: (i) experimental checks and calibrations in which the apparatus reproduces known phenomena or reproduces artifacts that are known in advance to be present; (ii) intervention, in which the experimenter manipulates the object under observation; (iii) independent confirmation using different experiments; (iv) elimination of plausible sources of error and alternative explanations of the result; (v) the use of the results themselves to argue for their validity; (vi) the use of an independently well-corroborated theory of the phenomena to explain the results; (vii) the use of an apparatus based on a wellcorroborated theory; and (viii) the use of statistical arguments.

Strategies such as these are embedded within a scientist's (narrow) perspective and can protect them against their subjective perspectives. Although such strategies do no entirely eliminate the threat of subjectivity or vicious instrumental theoryladeness, they go a long way in safeguarding instruments and their deliverances against this threat. And importantly, such strategies ensure that instruments can deliver objective empirical knowledge about the world.

Besides ensuring that scientists' subjective perspectives are kept in check, narrow perspectives play an additional role. Within a broad perspective, scientific disagreements, especially those concerning the results delivered by a scientific instrument, can occur more or less frequently. This may be because scientists who work within the same broad perspective, operate with the same objectivity standards, and work toward resolving the same empirical or conceptual problem may nonetheless have very different narrow perspectives. By identifying the differences among their assumptions, interests, or methods, disagreements can ultimately be resolved or dissolved (cf. Creţu 2020a). The distinction between "narrow perspectives" and "broad perspectives" thus not only facilitates the separation of distinct modalities in which instruments can be said to be perspectival, but it is also invaluable for understanding how and why disagreements can occur even when we hold broad perspectives and objectivity standards fixed. Conversely, the fact that scientists may disagree while sharing broad perspectives and objectivity standards, owing to their different narrow perspectives, offers a common basis for exploring a constructive resolution of the relevant disagreement.

Narrow perspectives and broad perspectives operate on different scales. Narrow perspectives concern the running of particular experiments, the operation of specific instruments, and the interpretation of specific data. In contrast, broad perspectives concern the development of a multitude of different narrow perspectives over a significant period of time. Narrow perspectives are more specialized and embed richer, more specific resources for justifying why we have good reasons to use a particular instrument, why the instrument is working properly, why the data it 
delivers are reliable, why disagreements occur, and how disagreements can be resolved or dissolved. And they can embed distinctively nonperspectival epistemic strategies to overcome instrumental theory-ladeness that are neither specific to a broad perspective nor governed by specific objectivity standards but are instead more broadly embedded within the epistemology of science. Thus, the epistemic checks that can ensure the reliability of instruments and their data are "internal" to neither a narrow perspective nor to a broad perspective; that is, if they are perspectival, they are so in a very broad sense.

To sum up, in this section, it has been shown that narrow perspectives have a dual nature in that they can both undermine and vindicate the use of instruments, the data they produce, and the knowledge engendered within both. To be precise, it was shown that when instruments are considered in their complexity, both on their own and within the web of experiments, there are many sources of theory input, many possibilities of error, many strategies for overcoming error, and many inputs from the phenomena or the world too. When we say that "instruments are perspectival," it must be clear what sort of dependence we have in mind and whether this is problematic or not. Merely identifying a generic skeptical worry does not, on its own, constitute a specific challenge to the epistemology of instruments and experiments.

\section{Conclusion and prospectus}

This article investigated the possible interpretations of the perspectivalist diagnosis that "instruments are perspectival." Some interpretations were found to be insightful but insufficiently developed, whereas others were identified with two well-known epistemic problems, conceptual relativism and theory-ladeness. Through the scholarship on objectivity and the history of canonical instruments (e.g., the cloud chamber), new perspectivalist solutions to the two well-known problems were developed. It was shown that conceptual relativism can be averted when objectivity standards governing broad perspectives do not change concomitantly with broad perspectives and when there is instrumental and data continuity from one broad perspective to the next and despite changing objectivity standards. Such continuity was demonstrated through the use of concrete examples drawn from the history of science. Regarding theory-ladeness, it was shown that this challenge is, at the same time, far more complex and far less problematic than previously assumed in relation to instruments. The novel response to this problem was to show that it can be more fruitfully analyzed in terms of narrow perspectives, which allow a closer examination of potential sources of justification and error and can constitute the key to understanding and resolving disagreements.

A compelling avenue for future research is to investigate the degree to which the described solutions bear out in realms of science increasingly dominated by the digital computer. Such a task would require, among other things, an extension of the scholarship on objectivity, the identification of clear-cut cases where the digital computer acts as an instrument, and a better understanding of the relationship between digital computers and broad and narrow perspectives. Further, it would be important to determine whether the digital computer is a natural extension of traditional instruments, leading to the same problems to which the same solutions 
apply, or whether new problems and solutions might require an even finer grain of perspectivism. ${ }^{21}$

Acknowledgments. I would like to thank two anonymous reviewers for their very helpful comments and for their time. I had very helpful conversations on this topic with Nicos Stylianou, James Ladyman, Karim Thébault, Vanessa Seifert, and Antonis Antoniou, to whom I am very grateful for their time. I would also like to thank Karim Thébault, Nicos Stylianou, Vanessa Seifert, and Antonis Antoniou for reading and commenting on several earlier drafts of this article. Samuel Fletcher and Michela Massimi have also kindly read and commented on an earlier version of this article. A big thanks also goes to Max Jones and to the Draft in Progress contingent at Bristol, Francesca Belazzi, David Cobb, Toby Friend, Richard Pettigrew, Oyku Ulusoy, and Lena Zuchowski. Work on this article has benefitted in part from funding from the European Research Council (ERC) under the European Union's Horizon 2020 research and innovation program (grant agreement European Consolidator Grant H2020-ERC2014-CoG 647272 Perspectival Realism. Science, Knowledge, and Truth from a Human Vantage Point).

\section{References}

Ackerman, Robert J. 1985. Data, Instruments, and Theory. A Dialectical Approach to Understanding Science. Princeton, NJ: Princeton University Press.

Avanzo, Salvatore, Roberto Barbera, Francesco De Mattia, Giuseppe La Rocca, Mariapaola Sorrentino, and Domenico Vicinanza. 2010. "The ASTRA (Ancient Instruments Sound/Timbre Reconstruction Application) Project Brings History to Life!" In Managed Grids and Cloud Systems in the Asia-Pacific Research Community, edited by Simon C. Lin and Eric Yen, 145-156. New York: Springer.

Baghramian, Maria, and J. Adam Carter. 2019. "Relativism." In Stanford Encyclopedia of Philosophy, edited by Edward N. Zalta. Stanford: Stanford University Press. https://plato.stanford.edu/entries/relativism/.

Baird, Davis. 2004. Thing Knowledge: A Philosophy of Scientific Instruments. Berkeley: University of California Press.

Baker, Kane. 2020. “Giere's Instrumental Perspectivism.” European Journal for Philosophy of Science 10 (3): 1-19.

Beauchemin, Pierre-Hugues. 2017. "Autopsy of Measurements with the ATLAS Detector at the LHC." Synthese 194 (2):275-312.

Blackett, Patrick Maynard Stuart. 1960. “Charles Thomson Rees Wilson, 1869-1959." Biographical Memoirs of Fellows of the Royal Society 6:269-95.

Bondi, Hermann. 1955. "Fact and Inference in Theory and in Observation." Vistas in Astronomy 1:155-62.

Brenni, Paolo. 2013. "From Workshop to the Factory: The Evolution of the Instrument-Making Industry, 1850-1930." In The Oxford Handbook of the History of Physics, edited by Jed Z. Buchwald and Robert Fox, 584-650. Oxford: Oxford University Press.

Brown, Harvey R. 2005. Physical Relativity. Space-Time Structure from a Dynamical Perspective. Oxford: Clarendon Press.

Chakravartty, Anjan. 2010. "Perspectivism, Inconsistent Models, and Contrastive Explanation." Studies in History and Philosophy of Science Part A 41 (4):405-12.

Chalmers, Alan. 2003. "The Theory-Dependence of the Use of Instruments in Science." Philosophy of Science 70 (3):493-509.

Chirimuuta, Mazviita. 2016. "Vision, Perspectivism, and Haptic Realism." Philosophy of Science 83 (5): $746-56$.

Creţu, Ana-Maria. 2020a. "Diagnosing Disagreements: The Authentication of the Positron 1931-1934." Studies in History and Philosophy of Science Part B: Studies in History and Philosophy of Modern Physics 70:28-38.

Creţu, Ana-Maria. 2020b. "Natural Kinds as Real Patterns: Or How to Solve the Commitment Problem for Perspectival Realism." Preprint, submitted January 18, 2020. http://philsci-archive.pitt.edu/16828/.

${ }^{21}$ The versatility of the digital computer (Parker 2010) is indicated by the fact that it can be involved not only in data production, but also in data analysis or the set-up of an experiment. Equally relevant is the complexity of computer simulations (Morgan and Morrison 1999; Humphreys 2004; Winsberg 2010; Morrison 2015). 
Creţu, Ana-Maria. 2020c. "Perspectival Realism." In Encyclopedia of Educational Philosophy and Theory, edited by Michael Adrian Peters, 23. Singapore: Springer.

Das Gupta, N. N., and S. K. Ghosh. 1946. "A Report on the Wilson Cloud Chamber and Its Applications in Physics." Reviews of Modern Physics 18 (2):225.

Daston, Lorraine, and Peter Galison. 2007. Objectivity. 2010 paperback ed. New York: Zone Books.

Evans, Peter W. 2020. "Perspectival Objectivity. Or: How I Learned to Stop Worrying and Love Observer-Dependent Reality." European Journal for Philosophy of Science 10 (2):1-21.

Franklin, Allan. 1986. The Neglect of Experiment. Cambridge: Cambridge University Press.

Franklin, Allan. 1989. "The Epistemology of Experiment." In The Uses of Experiment, edited by David Gooding, Trevor Pinch, and Simon Schaffer, 437-60. Cambridge: Cambridge University Press.

Franklin, Allan. 1990. Experiment, Right or Wrong. Cambridge: Cambridge University Press.

Franklin, Allan. 2015. "The Theory-Ladenness of Experiment." Journal for General Philosophy of Science 46 (1):155-66.

Franklin, Allan, M. Anderson, D. Brock, S. Coleman, J. Downing, A. Gruvander, J. Lilly et al. 1989. "Can a Theory-Laden Observation Test the Theory?" British Journal for the Philosophy of Science 40 (2):229-31.

Franklin, Allan, and Slobodan Perovic. 2019. "Experiment in Physics." In Stanford Encyclopedia of Philosophy, edited by Edward N. Zalta. Stanford: Stanford University Press. https://plato.stanford. edu/archives/win2019/entries/physics-experiment/.

Galison, Peter. 1997. Image and Logic: A Material Culture of Microphysics. Chicago: University of Chicago Press.

Giere, Ronald N. 2000. "The Perspectival Nature of Scientific Observation." Paper presented at the 17th Biennial Meeting of the Philosophy of Science (PSA 2000), Vancouver, British Columbia, Canada, November 2000. https://philsci.org/docs/perspectival-nature.pdf.

Giere, Ronald N. 1999. Science without Laws. Chicago: University of Chicago Press.

Giere, Ronald N. 2006. Scientific Perspectivism. 2010 paperback ed. Chicago: University of Chicago Press.

Giere, Ronald N. 2013. "Kuhn as Perspectival Realist." Topoi 32 (1):53-57.

Hacking, Ian. 1983. Representing and Intervening. Cambridge: Cambridge University Press.

Hackman, W. D. 1989. "Scientific Instruments: Models of Brass and Aids to Discovery." In The Uses of Experiment, edited by David Gooding, Trevor Pinch, and Simon Schaffer, 31-65. Cambridge: Cambridge University Press.

Hanson, Norwood Russell. 1958. Patterns of Discovery: An Inquiry into the Conceptual Foundations of Science. Cambridge: Cambridge University Press.

Hayton, Darin. 2012. "An Introduction to the Astrolabe." History of Science Pamphlets. https://dhayton. haverford.edu/wp-content/uploads/2012/02/Astrolabes.pdf.

Heidelberger, Michael. 2003. “Theory-Ladenness and Scientific Instruments in Experimentation.” In The Philosophy of Scientific Experimentation, edited by Hans Radder, 138-51. Pittsburgh: University of Pittsburgh Press.

Hicks, Mar. 2017. Programmed Inequality. How Britain Discarded Women Technologists and Lost Its Edge in Computing. 2018 paperback ed. Cambridge, MA: MIT Press.

Hoffleit, Dorrit. 1991. "The Evolution of the Henry Draper Memorial." Vistas in Astronomy 34 (Part 1): 107-62.

Humphreys, Paul. 2004. Extending Ourselves: Computational Science, Empiricism, and Scientific Method. Oxford: Oxford University Press.

Karaca, Koray. 2013. "The Strong and Weak Senses of Theory-Ladenness of Experimentation: Theory-Driven versus Exploratory Experiments in the History of High-Energy Particle Physics." Science in Context 26 (1):93-136.

King, David A. 1992. "Some Remarks on Islamic Astronomical Instruments." Scientiarum Historia: Tijdschrift voor de Geschiedenis van de Wetenschappen en de Geneeskunde 18 (1):5-23.

Kuhn, Thomas S. 1962. The Structure of Scientific Revolutions. Chicago: Chicago University Press.

Laudan, Larry. 1977. Progress and Its Problems: Towards a Theory of Scientific Growth. Berkeley: University of California Press.

Laudan, Larry. 1990. Science and Relativism: Some Key Controversies in the Philosophy of Science. Chicago: University of Chicago Press.

Lauwerys, Joseph Albert. 1937. "Scientific Instruments." Proceedings of the Aristotelian Society 38: 217-40. 
Light, Jennifer S. 1999. "When Computers Were Women." Technology and Culture 40 (3):455-83.

Massimi, Michela. 2012. "Scientific Perspectivism and Its Foes." Philosophica 84 (1):25-52.

Massimi, Michela. 2018a. "Four Kinds of Perspectival Truth." Philosophy and Phenomenological Research 96 (2):342-59.

Massimi, Michela. 2018b. "Perspectival Modeling." Philosophy of Science 85 (3):335-59.

Massimi, Michela. 2018c. "Perspectivism." In The Routledge Handbook of Scientific Realism, edited by Juha Saatsi, 164-75. New York: Routledge.

Massimi, Michela. 2018d. "Points of View: Kant on Perspectival Knowledge." Synthese 198 (3):3279-96.

Mayo, Deborah G. 1996. Error and the Growth of Experimental Knowledge. Chicago: University of Chicago Press.

McConnell, Anita. 2013. "Instruments and Instrument-Makers, 1700-1850." In The Oxford Handbook of the History of Physics, edited by Jed Z. Buchwald and Robert Fox, 326-57. Oxford: Oxford University Press.

Morgan, Mary S., and Margaret Morrison. 1999. Models as Mediators. Cambridge: Cambridge University Press.

Morgan, William Wilson, Philip C. Keenan, and Edith Kellman. 1943. An Atlas of Stellar Spectra. Chicago: University of Chicago Press.

Morrison, Margaret. 2011. "One Phenomenon, Many Models: Inconsistency and Complementarity." Studies in History and Philosophy of Science Part A 42 (2):342-51.

Morrison, Margaret. 2015. Reconstructing Reality: Models, Mathematics, and Simulations. Oxford Studies in Philosophy of Science. Oxford: Oxford University Press.

Parker, Wendy S. 2010. "An Instrument for What? Digital Computers, Simulation and Scientific Practice." Spontaneous Generations: A Journal for the History and Philosophy of Science 4 (1):39-44.

Roqué, Xavier. 1997. "The Manufacture of the Positron." Studies in History and Philosophy of Science Part B: Studies in History and Philosophy of Modern Physics 28 (1):73-129.

Schaffer, Simon. 2011. "Easily Cracked: Scientific Instruments in States of Disrepair." Isis 102 (4):706-17.

Schindler, Samuel. 2013. "Theory-Laden Experimentation." Studies in History and Philosophy of Science Part A 44 (1):89-101.

Staley, Kent W. 1999. “Golden Events and Statistics: What's Wrong with Galison's Image/Logic Distinction?" Perspectives on Science 7 (2):196-230.

Taub, Liba. 2019. "What Is a Scientific Instrument, Now?" Journal of the History of Collections 31 (3):453-67. Teller, Paul. 2011. "Two Models of Truth." Analysis 71 (3):465-72.

Turner, Anthony. 2013. "Physics and the Instrument-Makers, 1550-1700." In The Oxford Handbook of the History of Physics, edited by Jed Z. Buchwald and Robert Fox, 96-108. Oxford: Oxford University Press. Warner, Deborah Jean. 1990. "What Is a Scientific Instrument, When Did It Become One, and Why?" British Journal for the History of Science 23 (1):83-93.

Winsberg, Eric. 2010. Science in the Age of Computer Simulation. Chicago: University of Chicago Press.

Cite this article: Creţu, Ana-Maria. 2022. "Perspectival Instruments." Philosophy of Science 89 (3):521-541. https://doi.org/10.1017/psa.2021.32 